participants from the Alzheimer's Disease Neuroimaging Initiative (ADNI) with special emphasis on prodromal stages. Methods: 320 participants, including 106 healthy controls (HC), divided by genetic risk (family history of $\mathrm{AD}$ and/or $\mathrm{APOE} \varepsilon 4$ positive), 130 patients with early (EMCI) and 44 participants with late mild cognitive impairment (LMCI), and 29 patients with $\mathrm{AD}$, were included in this analysis. Florbetapir PET scans and structural MRI scans were downloaded from the ADNI site for the baseline Florbetapir visit and the two-year follow-up visit and processed using previously described techniques [1]. FDG PET scans were also downloaded from these timepoints and processed using standard techniques. Average Florbetapir SUVR, FDG SUVR, and structural grey matter density (GMD) were extracted from target ROIs at both visits, including the global cortex for the PET and bilateral mean hippocampus for MRI. Annualized percent change (APC) was calculated from these regions and compared between groups. Results: No group differences were observed for change in amyloid deposition. However, glucose metabolism decline and hippocampal GMD atrophy rate showed significant group differences $(\mathrm{p}<0.001$, Fig. 1A\&B). AD showed faster glucose decline and hippocampal atrophy than all other groups except LMCI $(\mathrm{p}<0.01)$. LMCI had a greater hippocampal atrophy rate than $\mathrm{HC}$ without risk and $\mathrm{EMCI}(\mathrm{p}<0.05)$. HC at genetic risk showed a non-significant trend toward greater decline in glucose metabolism and hippocampal GMD than $\mathrm{HC}$ without risk $(\mathrm{p}=0.1$ for GMD). Conclusions: Mean amyloid deposition does not appear to significantly change over two years in any stage of cognitive impairment. However, declining glucose metabolism and hippocampal atrophy rates appear to accelerate in later disease stages (LMCI, AD). Cognitively normal older adults at risk for $\mathrm{AD}$ due to genetic background may show greater rates of hippocampal atrophy and glucose metabolic decline than those without genetic risk but larger samples and replication are needed to further investigate this issue.[1] Risacher et al. (2013) Front Aging Neurosci
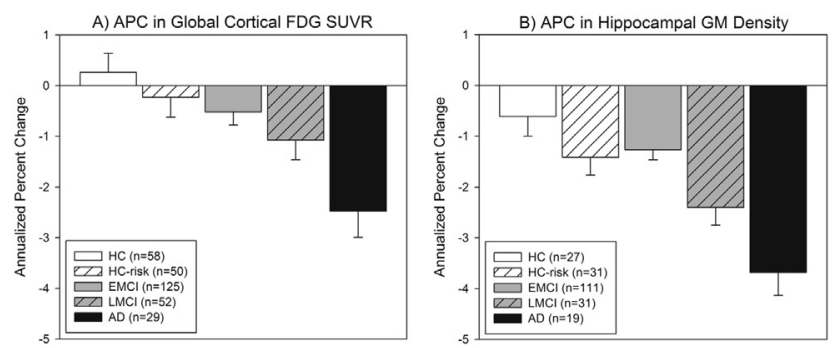

Figure 1. APC in Glucose Metabolism and Hippocampal GM Density

\section{IC-P-096 INCREASED AMYLOID DEPOSITION IN OLDER ADULTS AT RISK FOR PROGRESSION TO ALZHEIMER'S DISEASE DUE TO GENETIC BACKGROUND AND/OR THE PRESENCE OF SIGNIFICANT MEMORY CONCERNS}

Shannon Leigh Risacher ${ }^{1}$, Sungeun $\mathrm{Kim}^{1}$, Kwangsik T. Nho ${ }^{1}$, John D. West ${ }^{1}$, Yang Wang ${ }^{1}$, Ronald Carl Petersen ${ }^{2}$, Paul S. Aisen ${ }^{3}$, Clifford R. Jack ${ }^{4}$, William J. Jagust ${ }^{5}$, Robert Koeppe ${ }^{6}$,

Michael Walter Weiner ${ }^{7}$, Andrew J. Saykin ${ }^{8},{ }^{1}$ Indiana University School of Medicine, Indianapolis, Indiana, United States; ${ }^{2}$ Mayo Clinic Rochester, Rochester, Minnesota, United States; ${ }^{3}$ UCSD, La Jolla, California, United States; ${ }^{4}$ Mayo Clinic, Rochester, Minnesota, United States; ${ }^{5}$ University of California, Berkeley, Berkeley, California, United States; ${ }^{6}$ University of Michigan, Ann Arbor, Michigan, United States; ${ }^{7}$ Center for Imaging of Neurodegenerative Diseases; VA Medical Center and UCSF, San Francisco, United States; ${ }^{8}$ Indiana University School of
Medicine, Indianapolis, Indiana, United States.

Contact e-mail: srisache@iupui.edu

Background: Older adults with significant memory concerns (SMC) and/or genetic risk for $\mathrm{AD}$ are key groups of interest due to risk of progression. Our goal was to evaluate amyloid deposition, glucose metabolism, and medial temporal lobe (MTL) atrophy in SMC participants from ADNI. Methods: 569 participants were selected from the ADNI cohort, including 177 healthy controls (HC), 93 participants with SMC, and 299 patients with early mild cognitive impairment (EMCI). The HC participants were further divided into those with genetic risk (APOE $\varepsilon 4$ positive and/or family history of $\mathrm{AD}$ (HC-risk)) and those without genetic risk (HC). The SMC participants were also further divided by the presence or absence of informant complaints about the participant's memory. SMC participants were considered to have self-only concerns (SMC) if the informant did not endorse $>1.5$ SD above the HC mean on the ECog Memory domain ( $\sim 62 \%$ of items) and to have self-plus-informant concerns if the informant endorsed $>62 \%$ (SMC-plus). Florbetapir and structural MRI scans were downloaded from the ADNI site for the baseline visit and processed as previously described [1]. FDG PET scans were also downloaded from this timepoint and processed using standard techniques. Average Florbetapir SUVR, FDG SUVR, and structural grey matter density were extracted from the global cortex (PET) and bilateral hippocampus (MRI). Florbetapir PET scans were also compared between groups on a voxel-wise level in SPM8. Results: A significant difference in amyloid deposition was observed between groups on both voxel-wise and ROI analyses. HC-risk, SMC, and SMC-plus demonstrated more global and regional amyloid deposition than $\mathrm{HC}$ without risk, including in the global cortex $(\mathrm{p}=0.004)$ and precuneus $(\mathrm{p}<0.001)$. Differences in glucose metabolism and MTL atrophy were more variable with some regions showing a trend towards hypometabolism and MTL atrophy in HC-risk and SMC groups. Conclusions: Participants with SMC and $\mathrm{HC}$ participants at risk for progression to $\mathrm{AD}$ due to genetic background show increased amyloid deposition relative to $\mathrm{HC}$ without risk. This suggests that older adults with SMC, especially those with informant corroboration, are at increased risk for future cognitive decline and therefore may be a good target population for enrichment of clinical trials.[1] Risacher et al. (2013) Frontiers in Aging Neuroscience.
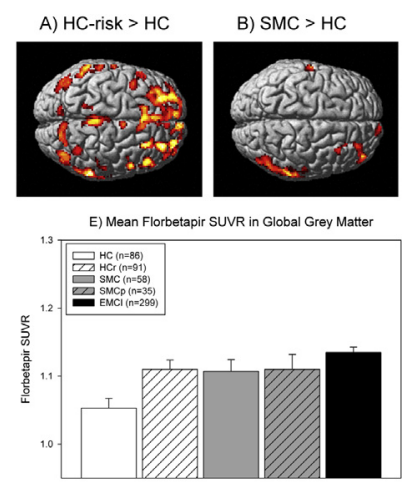

Figure 1. Increased Amyloid Deposition in Older Adults at Risk for AD
E) Mean Florbetapir SUVR in Global Grey Matter

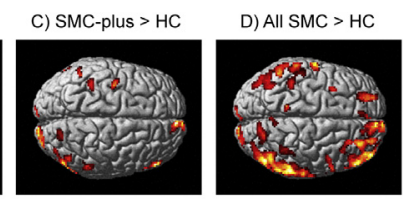

F) Mean Florbetapir SUVR in Bilateral Precuneus

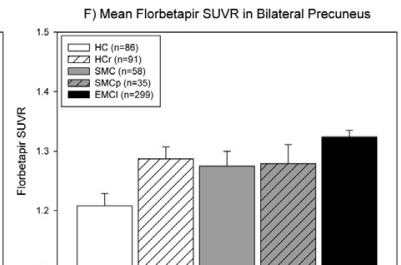

IC-P-097 VISUAL AND AUDITORY CHANGES ARE ASSOCIATED WITH NEUROIMAGING BIOMARKERS DURING PRODROMAL STAGES OF ALZHEIMER'S DISEASE

Shannon Leigh Risacher ${ }^{1}$, Darrell WuDunn ${ }^{1}$, Karmen Yoder ${ }^{1}$, John West ${ }^{1}$, Brenna C. McDonald ${ }^{1}$, Eileen M. Tallman ${ }^{1}$, Yang Wang ${ }^{1}$, Sujuan Gao ${ }^{1}$, Martin Rhys Farlow ${ }^{1}$, Andrew J. Saykin ${ }^{2},{ }^{1}$ Indiana University School of 